\title{
Obtención y caracterización de fibras vegetales para la mejora nutricional de caramelos tipo gummies: avances de tesis doctoral
}

\section{Obtaining and characterization of vegetable fibers for the nutritional improvement of gummy candies: doctoral thesis progress}

Presentación: 6-7/10/2020

Doctorando:

Cecilia Gabriela Giménez

Grupo de Investigación en Biotecnología y Alimentos (BIOTEC), Universidad Tecnológica Nacional, Facultad Regional Resistencia - Argentina. Ceciliagimenez25@gfe.frre.utn.edu.ar

Director/es:

Silvia Del Carmen Rodríguez

Co-director/es:

Carola Andrea Sosa

\section{Resumen}

Considerando que el sobrepeso es una consecuencia de una malnutrición, que afecta a todos los países de la región y tiene alcance global, es importante mantener una buena alimentación mediante el consumo de alimentos saludables y poco calóricos. Consecuentemente, las investigaciones del área de alimentos se orientan a obtener ingredientes con características funcionales a partir de fuentes naturales, para incorporarlos en alimentos que propicien una dieta balanceada y nutritiva, y que prevengan enfermedades crónicas. Uno de estos componentes funcionales es la fibra dietaria, que se encuentra en una gran variedad de hortalizas como las mandiocas, batatas, y jengibres, cultivados en el Nordeste argentino. Por consiguiente, este plan de tesis doctoral consiste en la reformulación de caramelos tipo gummies con el objeto de mejorarlos nutricionalmente a partir de la adición de fibra extraída de vegetales regionales. La misma actuará, además, como agente estructural debido al reemplazo de los azúcares presentes en la formulación por edulcorantes no calóricos con el fin de reducir del aporte calórico.

En este trabajo, se presentarán los avances realizados del mencionado plan, que comenzó a mediados del año 2019 y continua hasta la actualidad. Primeramente, se realizó el ensayo de extracción de fibra dietaria de batata utilizando un método tradicional químico con etanol al $85 \%$ (v/v) como solvente, y se determinó el rendimiento. Se definieron los parámetros del proceso tales como las condiciones de pre-tratamiento, temperaturas de extracción y secado, ratio, tiempo de secado y tamaño de tamiz, dando como resultado rendimientos de $10 \%$ aproximadamente. Además, se llevaron a cabo las determinaciones de los componentes bioactivos: carotenoides totales y capacidad antioxidante de las materias primas batata, jengibre y mandioca, con el fin de caracterizarlas. En ambos casos se utilizaron métodos espectrofotométricos, y se obtuvieron valores del contenido de carotenoides totales de $14.27 \pm 0.55 \mu \mathrm{g}_{\beta \text {-caroteno }} / \mathrm{g}_{\text {muestra }}$ para el jengibre, y de $12.66 \pm 1.43$ y $1.35 \pm 0.06 \mu \mathrm{g}_{\beta \text {-caroteno }} / \mathrm{g}_{\text {muestra, }}$ para la batata y mandioca. Un comportamiento similar ocurrió en el caso de la capacidad antioxidante donde el jengibre fue un $90 \%$ y $50 \%$ mayor en comparación con la mandioca y la batata, respectivamente.

De este modo, se determinó el rendimiento de la extracción química de fibra a partir de batata, y se observó que el jengibre fue el material vegetal con mayor contenido de compuestos bioactivos, lo que lo hace un ingrediente potencialmente utilizable para lograr la mejora nutricional de los alimentos.

Palabras clave: Fibra dietaria, extracción-caracterización, caramelos saludables 


\begin{abstract}
Considering that being overweight is a consequence of malnutrition, that affects all the countries in this region and has also a global reach, it is important to maintain a good diet by eating healthy and low-calorie foods. Consequently, researchs in the food area are aimed to obtain ingredients with functional characteristics from natural sources, to incorporate them into foods that promote a balanced and nutritious diet, and therefore, prevent chronic diseases. One of these functional components is dietary fiber, which is found in a wide variety of vegetables such as cassava, sweet potatoes, and gingers, grown in the Northeast of Argentina. Consequently, this doctoral thesis plan propouses the reformulation of gummies type candies to improve them nutritionally with the addition of fiber extracted from regional vegetables. It will also act as a structural agent due to the replace the sugars present in the formulation with non-caloric sweeteners to reduce the caloric intake.

In this work, the progress made from the plan will be presented, which began in mid-2019 and continues until today. Firstly, the sweet potato dietary fiber extraction test was performed by using a traditional chemical method with ethanol at $85 \%$ (v / v) as solvent, and the yield was determined. Process parameters such as pre-treatment conditions, extraction and drying temperatures, ratio, drying time, and sieve size were defined, resulting in yields of $10 \%$ approximately. In addition, determinations of the bioactive components were carried out: total carotenoids and antioxidant capacity of the raw material's sweet potato, ginger, and cassava, to characterize them. In both cases, spectrophotometric methods were used, and total carotenoid content values of $14.27 \pm 0.55 \mu \mathrm{g}_{\beta}$-carotene $/ \mathrm{g}_{\text {sample }}$ were obtained for ginger, and of $12.66 \pm 1.43$ and $1.35 \pm 0.06 \mu \mathrm{g}_{\beta \text {-carotene }} / \mathrm{g}_{\text {sample, }}$ for sweet potato and cassava. A similar behavior occurred in the case of antioxidant capacity where ginger was $90 \%$ and $50 \%$ higher compared to cassava and sweet potato, respectively.

In this way, the performance of the chemical extraction of fiber from sweet potatoes was determined, and it was observed that ginger was the plant material with the highest content of bioactive compounds, which makes it a potentially usable ingredient to achieve the nutritional improvement of foods.
\end{abstract}

Keywords: Dietary fiber, extraction-characterization, healthy candies

\title{
Introducción
}

En Argentina, el exceso de peso afecta al 13,6 \% de la población menor a 5 años, mientras que el 1,7 \% presenta bajo peso y el 1,6\% padece desnutrición aguda. En el caso de los niños y adolescentes de 5 a 17 años, el 20,7 \% tiene sobrepeso y el 20,4\%, obesidad. En cuanto a los mayores de 18 años, el $34 \%$ tiene sobrepeso y la obesidad afecta al 33,9 \% (ENNyS/SGS/MSDS, 2019). Ante este panorama, la calidad nutricional de las golosinas se ubica cada vez más en el punto de mira de los consumidores que manifiestan un mayor interés hacia opciones más saludables y naturales.

La fibra dietaria (FD) es uno de los componentes funcionales importantes, tanto por los efectos benéficos en la salud tales como reforzar el sistema inmune, absorber grasa, controlar el peso corporal y prevenir la obesidad; como por las aplicaciones en la industria alimentaria, desde un punto de vista tecnológico (Huang et al., 2018). Es así como su incorporación a confituras o golosinas elaboradas con vegetales y frutas, fortificadas y de bajo contenido calórico, podría contribuir al cambio y la mejora de las características sensoriales y texturales, por sus propiedades tecnológicas como el aumento de la capacidad de retención de agua y aceite, la modificación de la textura, la estabilización de la estructura, efectos fisiológicos y la extensión de la vida útil (Tejada-Origoza et al., 2016).

El concepto de fibra dietaria (FD) fue cambiando a lo largo del tiempo. Inicialmente Hipsley et al. (1953), la definieron como los componentes no digeribles de las paredes celulares de los vegetales, es decir, hemicelulosa, celulosa y lignina. Durante la década de los 70s se definió a la FD como los restos de células vegetales resistentes a la hidrólisis (digestión) llevada a cabo por las enzimas alimentarias del hombre; considerándose como restos de células a los polisacáridos de origen vegetal constituidos por celulosa, hemicelulosa y lignina (Burkitt et al., 1972). Recién en 1985 se incluye a los oligosacáridos, pectinas, gomas y ceras (Towel et al., 1985). En el 2009, el CODEX Alimentarius incluyó a los polímeros no digeribles de 3 a 9 grados de polimerización, tales como la insulina, maltodextrinas resistentes, rafinosa y otros. También ha reconocido a la lignina y otros componentes (polifenoles, ceras, saponinas, fitatos, cutinas y fitoesteroles) como parte de la FD, siempre que estén asociados a los componentes de la pared celular vegetal (McCleary et al., 2010).

La primera etapa en el proceso de obtención de fibra es la remoción de la matriz en la que se encuentra. En este sentido, el factor más importante es la optimización de las condiciones de extracción, ya que afectan a las propiedades funcionales y químicas, composición y solubilidad. Las extracciones pueden ser realizadas por técnicas 
convencionales como las extracciones térmicas, químicas y enzimáticas, o mediante la utilización de tecnologías verdes como es el ultrasonido o las microondas, las cuales pueden dan lugar a diferentes propiedades fisicoquímicas, funcionales y estructurales de la fibra. Asimismo, el procesamiento a alta presión es una tecnología utilizada recientemente para la mejora de la extracción, con resultados prometedores sobre las mencionadas propiedades (Mateos-Aparicios et al., 2010).

Esta FD se encuentra en una gran variedad de fuentes naturales desde cascarilla de arroz hasta hortalizas y vegetales de diversos tipos. En el Nordeste Argentino (NEA), integrado por las provincias de Corrientes, Chaco, Formosa y Misiones, se cultivan hortalizas que pueden ser potenciales fuentes de fibra como el caso de la mandioca (Manihot esculenta Crantz), batata (Ipomoea batatas (L.), y jengibre (Zingiber officinalis). Asimismo, el procesamiento de éstas como fuentes de ingredientes funcionales, constituye una posibilidad para el aprovechamiento de la producción, así como para obtener productos alimenticios de mayor valor agregado.

\section{Desarrollo}

Se acepta la inscripción definitiva a la carrera de Doctorado en Ciencias y Tecnologías de Alimentos, con orientación Ingeniería a principios de julio del 2019, y las actividades están planteadas para cinco años (quince cuatrimestres). Las que corresponden a los primeros tres cuatrimestres tienen que ver con métodos de extracción de fibra dietaria, y a partir del tercero y hasta el quinto, con cuantificación de la fibra por métodos enzimáticos. Asimismo, la revisión bibliográfica se realiza a lo largo de todo el proceso de formación.

\section{Materiales y métodos}

\section{- Extracción química de fibra dietaria procedente de batata}

Se diagramó un esquema de proceso de extracción a seguir, utilizando un método tradicional químico donde se pone en contacto a la batata con un solvente polar (etanol al $80 \%(\mathrm{v} / \mathrm{v})$ ). Se establecieron una serie de parámetros de extracción: pre-tratamiento de la materia prima, solvente, temperatura de extracción, ratio, temperatura de secado, tiempo de secado y tamaño de tamiz; y se realizaron cuatro extracciones con Soxhlet. Luego, el extracto obtenido, se secó mediante secado por convección de aire a $50^{\circ} \mathrm{C}$ durante 24 horas y se trituró para obtener un polvo de fibra. Las extracciones fueron evaluadas en primera instancia en función del rendimiento.

- Determinación de los compuestos bioactivos de las materias primas: mandioca, batata y jengibre

La presencia de compuestos bioactivos en los alimentos contribuye a definir las características organolépticas y a preservar la calidad nutricional, además de ejercer un efecto positivo en la salud de los consumidores, como prevenir el desarrollo de enfermedades crónicas-degenerativas que limitan la calidad y expectativa de vida de la población mundial (Giménez et al.; 2018). Consecuentemente, la tendencia a consumir este tipo de alimentos se encuentra en constante crecimiento.

Se realizaron determinaciones de compuestos bioactivos: carotenoides totales y capacidad antioxidante total, de las fuentes de fibra dietaria (mandioca, batata y jengibre), con el objeto de caracterizarlas.

Para la determinación de carotenoides totales, se pesaron $2 \mathrm{~g}$ de cada matriz a analizar (mandioca, batata y jengibre), se molturaron con $5 \mathrm{~mL}$ de acetona y se filtró a vacío, con enjuague hasta decoloración total. Se registró el volumen de extracto acetónico y una alícuota del mismo se mezcló con partes iguales de éter de petróleo. Luego, se separaron las fases en una ampolla de decantación, recolectando la parte superior. Finalmente, se realizaron las lecturas de absorbancia a $\lambda=450 \mathrm{~nm}$ (contra un blanco de éter de petróleo), en un espectrofotómetro marca PERKIN ELMER LAMBDA $25 \mathrm{UV}$-visible. Los carotenoides totales se expresaron como $\left[\mu \mathrm{g}_{\beta}\right.$-caroteno$\left._{\mathrm{g}} / \mathrm{g}_{\mathrm{muestra}}\right]$. Los análisis se realizaron por triplicado.

La determinación de capacidad antioxidante se llevó a cabo mediante el radical cromóforo DPHH $\bullet$. Para ello, se pesaron 0,5 g de cada matriz a analizar (mandioca, batata y jengibre); se molturaron con $3 \mathrm{~mL}$ de etanol y se filtraron a vacío. Posteriormente, se tomaron alícuotas de $0,5 \mathrm{~mL}$ del filtrado y se hicieron reaccionar con $6 \mathrm{~mL}$ de una solución de DPPH• $(0,003 \mathrm{~g} / 100 \mathrm{~mL}$ de etanol). Finalmente, se colocaron en oscuridad durante 20 minutos y se realizaron las lecturas de absorbancia a $\lambda=517 \mathrm{~nm}$ (usando etanol como blanco), en un espectrofotómetro marca PERKIN ELMER LAMBDA 25 UV-visible. La curva de calibración se realizó con soluciones seriadas de Trolox (2 $\mathrm{mg} / 10 \mathrm{~mL}$ de metanol), con los cuales se realizó el mismo procedimiento. Los resultados se expresaron como $\left[\mathrm{mg}_{\text {Trolox EQ}} / \mathrm{g}_{\text {muestra] }}\right]$. 


\section{Resultados}

Los resultados que se obtuvieron se muestran a continuación.

- Extracción química de fibra dietaria procedente de batata

En la Tabla 1, se detallan los parámetros establecidos en el proceso de extracción química de FD, utilizando etanol al $85 \%$ (v/v) como solvente de extracción y tubérculos de batata como materia prima. Se puede observar que los rendimientos que se obtuvieron fueron del $10,36 \%$ en promedio.

\begin{tabular}{|c|c|c|c|c|c|}
\hline Condiciones de extracción Método 1 & $\begin{array}{c}\text { Masa de } \\
\text { muestra [g] }\end{array}$ & $\begin{array}{c}\text { Masa de fibra } \\
\text { obtenida }[\mathrm{g}]\end{array}$ & $\begin{array}{c}\text { Masa de } \\
\text { fibra seca [g] }\end{array}$ & $\begin{array}{c}\text { Rendimiento } \\
{[\%]}\end{array}$ & $\begin{array}{c}\text { Humedad } \\
{[\%]}\end{array}$ \\
\hline \multirow{4}{*}{$\begin{array}{c}\text { Matriz: Batata. } \\
\text { Pretratamiento: Escaldado } 45 \mathrm{~min} \text {. } \\
\text { Solvente: Etanol } 85 \%(\mathrm{v} / \mathrm{v}) . \\
\text { Temperatura de extracción: } 80^{\circ} \mathrm{C} \text {. } \\
\text { Ratio: } 1 / 10 . \\
\text { Temperatura de secado: } 50^{\circ} \mathrm{C} \text {. } \\
\text { Tiempo de secado: } 24 \mathrm{~h} . \\
\text { Tamaño tamiz: malla } 80 .\end{array}$} & 10 & 1,0385 & 1,0161 & 10,39 & 2,16 \\
\hline & 25 & 2,7827 & 2,7083 & 11,13 & 2,67 \\
\hline & 30 & 3,0502 & 2,9839 & 10,17 & 2,17 \\
\hline & 30 & 2,9244 & 2,8525 & 9,75 & 2,46 \\
\hline
\end{tabular}

Tabla 1: Resultados del Método 1- Extracción con Soxhlet-Metanol 85\%

- Determinación de los compuestos bioactivos de las materias primas: mandioca, batata y jengibre

En las Figuras 1A y 1B, se observan los valores de capacidad antioxidante y contenido de carotenoides totales de los vegetales estudiados.

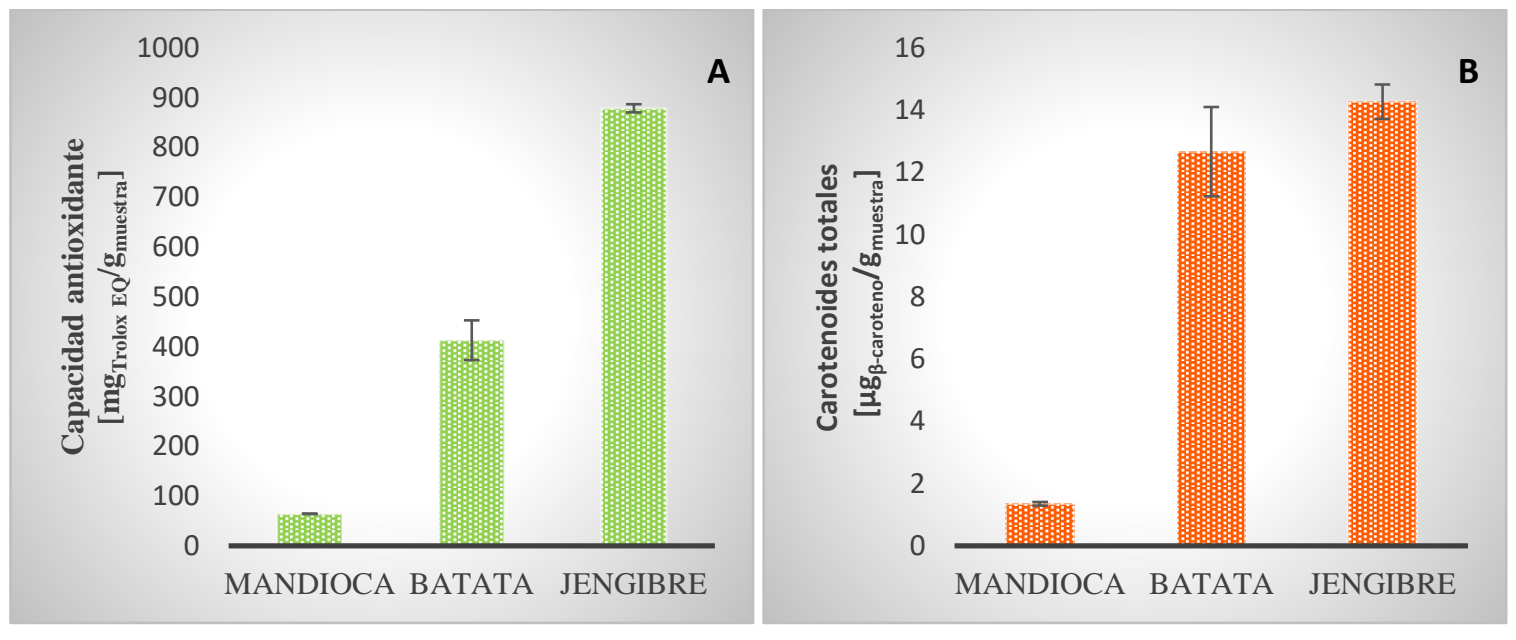

Figura 1. (A) Capacidad antioxidante total $\left[\mathrm{mg}_{\text {Trolox EQ }} / \mathrm{g}_{\text {muestra }}\right]$; (B) Carotenoides totales $\left[\mu \mathrm{g}_{\beta \text {-caroteno }} / \mathrm{g}_{\text {muestra }}\right]$; presentes en mandioca, batata y jengibre.

Los resultados mostraron que el jengibre presentó una capacidad antioxidante un $90 \%$ y $50 \%$ mayor en comparación con la mandioca y la batata, respectivamente. Coincidentemente a lo observado, el contenido de carotenoides totales en esta hortaliza fue de $14.27 \pm 0.55 \mu \mathrm{g}_{\beta}$-caroteno/gmuestra, mayor a los encontrados para batata $\mathrm{y}$ mandioca (12.66 \pm 1.43 y $1.35 \pm 0.06 \mu \mathrm{g}_{\beta \text {-caroteno/gmuestra }}$ respectivamente).

Similarmente, De Lima et al. (2017) registraron un elevado contenido de compuestos bioactivos y capacidad antioxidante en jengibre. Por su parte, Tang et al. (2015), y Koch et al. (2016), llevaron a cabo determinaciones en mandioca y batata, obteniendo para estos vegetales valores similares a los de este estudio. 


\section{Conclusiones}

La incorporación de ingredientes funcionales a los alimentos, como es el caso de la fibra dietaria, es un factor de importancia al considerar la mejora nutricional y reducción del aporte calórico de los mismos.

Los resultados de los ensayos realizados en el marco del plan de tesis mostraron la factibilidad de obtención de FD por medio de un método químico tradicional como es la extracción con solventes, a partir de batata, un vegetal altamente disponible en nuestra región. Además, y desde el punto de vista nutricional, todas las materias primas que se pretenden emplear como potenciales fuentes de fibra, mostraron un contenido apreciable en compuestos bioactivos como antioxidantes y carotenoides.

Cabe destacar que, las actividades experimentales que hubieran permitido presentar más avances, se vieron afectadas por la pandemia del COVID-19, que impidió la asistencia a los laboratorios y lugares de trabajo.

Asimismo, se prosiguió con la actualización bibliográfica, presentación de artículos en congresos y seminarios de la especialidad, tanto nacionales como internacionales, y se pretende capitalizar el material leído con la presentación de un artículo de revisión en una revista de alto impacto.

Como actividades futuras, se plantea continuar con los ensayos de extracción de FD de jengibre y mandioca, y evaluar los otros métodos de extracción tradicionales, así como los emergentes, con la finalidad de encontrar las condiciones óptimas. Además, realizar la caracterización fisicoquímica, las determinaciones de las propiedades fisiológicas y tecnológicas, y la incorporación de la fibra a alimentos confituras.

\section{Referencias}

Burkitt, D. P., Walker, A. R. P., \& Painter, N. S. (1972). Effect of dietary fibre on stools and transit-times, and its role in the causation of disease. The Lancet, 300(7792), 1408-1411.

De Lima, A. C. S., da Rocha Viana, J. D., de Sousa Sabino, L. B., da Silva, L. M. R., da Silva, N. K. V., \& de Sousa, P. H. M. (2017). Processing of three different cooking methods of cassava: Effects on in vitro bioaccessibility of phenolic compounds and antioxidant activity. LWT-Food Science and Technology, 76, 253-258.

Encuesta Nacional de Nutrición y Salud. Secretaría de Gobierno. Ministerio de Salud y Desarrollo Social. (2019). $2^{\circ}$ Encuesta Nacional de Nutrición y Salud. Recuperado de http://www.msal.gob.ar/images/stories/bes/graficos/0000001602cnt-2019-10 encuesta-nacional-de-nutricion-y$\underline{\text { salud.pdf }}$

Hipsley, E. H. (1953). Dietary “fibre” and pregnancy toxaemia. British medical journal, 2(4833), 420.

Huang, L., Zhang, X., Xu, M., An, S., Li, C., Huang, C., ... \& Liu, Y. (2018). Dietary fibres from cassava residue: Physicochemical and enzymatic improvement, structure and physical properties. AIP Advances, 8(10), 105035.

Mateos-Aparicio, I., Mateos-Peinado, C., \& Rupérez, P. (2010). High hydrostatic pressure improves the functionality of dietary fibre in okara by-product from soybean. Innovative Food Science \& Emerging Technologies, 11(3), 445450.

McCleary, B. V., De Vries, J. W., Rader, J. I., Cohen, G., Prosky, L., Mugford, D. C., ... \& Okuma, K. (2010). Determination of total dietary fiber (CODEX definition) by enzymatic-gravimetric method and liquid chromatography: Collaborative study. Journal of AOAC international, 93(1), 221-233.

Scafuri, B., Marabotti, A., Carbone, V., Minasi, P., Dotolo, S., \& Facchiano, A. (2016). A theoretical study on predicted protein targets of apple polyphenols and possible mechanisms of chemoprevention in colorectal cancer. Scientific reports, 6(1), 1-13.

Tang, Y., Cai, W., \& Xu, B. (2015). Profiles of phenolics, carotenoids and antioxidative capacities of thermal processed white, yellow, orange, and purple sweet potatoes grown in Guilin, China. Food Science and Human Wellness, 4(3), 123-132.

Tejada-Ortigoza, V., Garcia-Amezquita, L. E., Serna-Saldívar, S. O., \& Welti-Chanes, J. (2016). Advances in the functional characterization and extraction processes of dietary fiber. Food Engineering Reviews, 8(3), 251-271. 
Trowell, H., Burkitt, D., \& Heaton, K. (1985). Dietary fibre, fibre-depleted foods and disease. 